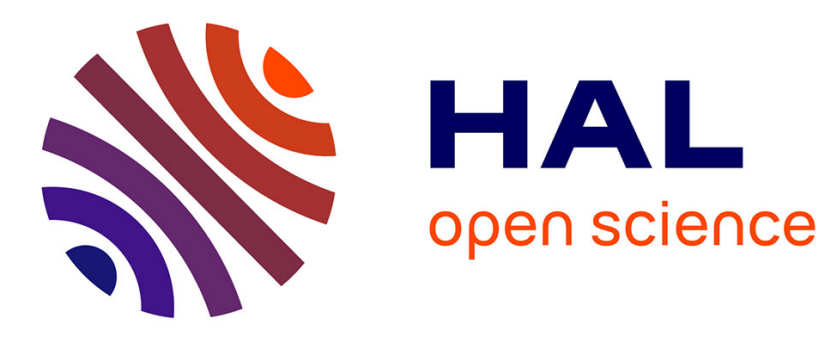

\title{
Review of materials science and supercritical fluids
}

François Cansell, Cyril Aymonier, Anne Loppinet-Serani

\section{To cite this version:}

François Cansell, Cyril Aymonier, Anne Loppinet-Serani. Review of materials science and supercritical fluids. Current Opinion in Solid State and Materials Science, 2003, 7 (4-5), pp.331-340. 10.1016/j.cossms.2004.01.003 . hal-00165780

\section{HAL Id: hal-00165780 \\ https://hal.science/hal-00165780}

Submitted on 8 Jun 2021

HAL is a multi-disciplinary open access archive for the deposit and dissemination of scientific research documents, whether they are published or not. The documents may come from teaching and research institutions in France or abroad, or from public or private research centers.
L'archive ouverte pluridisciplinaire HAL, est destinée au dépôt et à la diffusion de documents scientifiques de niveau recherche, publiés ou non, émanant des établissements d'enseignement et de recherche français ou étrangers, des laboratoires publics ou privés. 


\title{
Review on materials science and supercritical fluids
}

\author{
François Cansell *, Cyril Aymonier, Anne Loppinet-Serani \\ Institut de Chimie et de la Matière Condensée de Bordeaux (I.C.M.C.B), Université Bordeaux 1, 87, Avenue Albert Schweitzer, \\ 33608 Pessac Cedex, France
}

\begin{abstract}
Some supercritical fluids are able to replace toxic industrial solvents. So, from an industrial point of view, supercritical fluids are widely used in many fields such as pharmacy, food industry and environment. From a scientific point of view, the main interest of supercritical fluids is connected to the possibility to adjust continuously the physicochemical properties of these reactive media, such as selectivity, solvation, solubility or reactivity. In this paper, we present recent work on processes using supercritical media for processing materials related to fine particle synthesis, porous materials synthesis, polymer synthesis and surface modifications.
\end{abstract}

Keywords: Material; Supercritical fluid; Nanomaterial; Particle; Polymer; Cleaning; Surface modification; Porous material

\section{Introduction}

Both the capability of some supercritical fluids to replace toxic solvents and the ability of tuning solvent characteristics for highly specific separations or reactions have led to the current scientific and industrial interest in supercritical fluids. These fluids possess physicochemical properties, such as density, viscosity and diffusivity, which are intermediate between those of liquids and gases and which are continuously adjustable from gas to liquid with small pressure and temperature variations. Processes using these fluids are particularly attractive in many fields such as analytical and preparative separations, organic and inorganic synthesis, waste management, material processing (nanomaterials, nanostructured materials, thin films, coating), porous materials and earth science (volcanism, geothermal energy, hydrothermal synthesis) $[* 1, * 2, * 3]$.

A fluid is said to be "supercritical" when its temperature and pressure exceed the critical temperature $\left(T_{\mathrm{c}}\right)$ and pressure $\left(P_{\mathrm{c}}\right)$. Fig. 1 shows a schematic representation of density and organization of molecules of a pure fluid in solid state, gas state, liquid state and supercrit-

\footnotetext{
${ }^{*}$ Corresponding author. Tel.: $+33540002673 / 556842673$; fax: +33540002761/556842761.

E mail address: cansell@icmcb.u bordeaux.fr (F. Cansell).
}

ical domain. At the critical point, the density of the gas phase becomes equal to that of the liquid phase and the interface between gas and liquid disappears. The macroscopic behaviour of these fluids is characterized by mechanical and thermal instabilities. These instabilities result from the singular behaviour of some properties such as the isothermal compressibility $\left(K_{\mathrm{T}}=-1 /\right.$ $V(\mathrm{~d} V / \mathrm{d} P)$ where $V$ is the molar volume), which tends to infinity. The value of this isothermal compressibility remains important in the current conditions of using supercritical fluids $\left(1<\frac{T}{T_{\mathrm{c}}}<2\right.$ and $\left.1<\frac{P}{P_{\mathrm{c}}}<3\right)$. From a microscopic point of view, this behaviour has been interpreted by local fluctuations of the local density leading to the critical opalescence phenomenon due to the fact that the correlation length is of the same order of magnitude that of visible light. As reported in Fig. 2a, the local organization includes simultaneously regions of both gas and liquid local densities. This local organization is dynamic (with a life time of approximately $100 \mathrm{ps}$ ) and these local fluctuations of density allow us to explain the increase of the fluid compressibility. In order to discuss this point, we will study the case of a highpressure vessel with a volume $V$ and filled with $n$ molecules at $T$ temperature.

In this high-pressure vessel, the fluid can be described as a system consisting of microdomains with localized densities of both gaseous and liquid levels which are in dynamic equilibrium. If one reduces the volume $V$ (with $T$ and $n$ constant) with a mechanical action, the system 


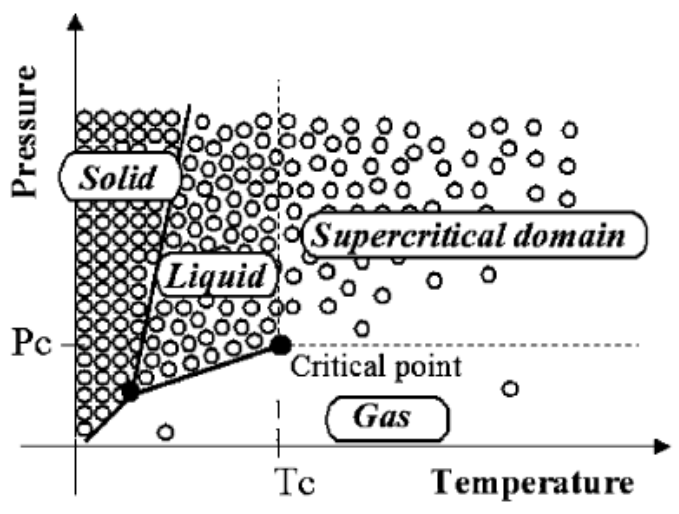

Fig. 1. Schematic representation of microscopic behaviour of a pure fluid in the $P T$ plane phase diagram.
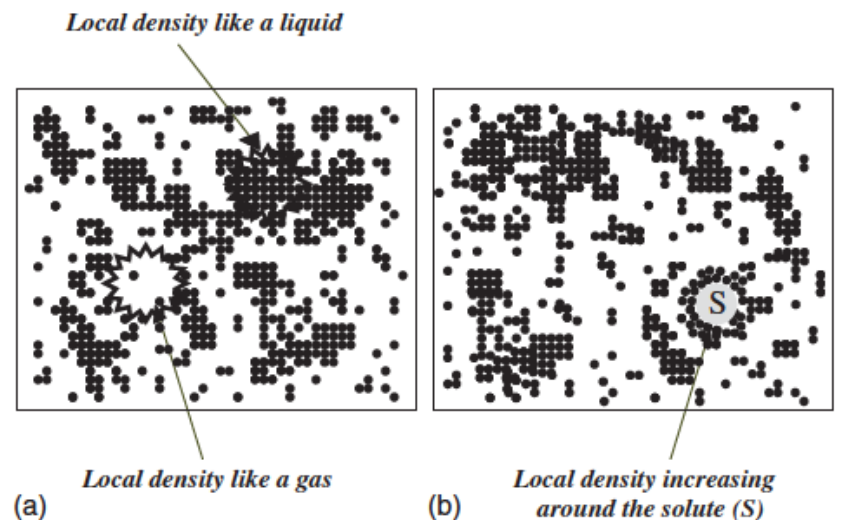

Fig. 2. Schematic representation of the molecule distribution simula tion in a supercritical pure fluid (a) and solvent and solute molecules in a supercritical solution (b).

will react and particles in a microdomain with a local density like a gas will go to a microdomain with a local density like a liquid. So this reaction of the system leads to a very weak increasing of pressure and explains the high value of the fluid compressibility.

Regarding the classical description of solvation, this takes into account interactions at small distances whereas interactions at long distances, due to the critical behaviour of the fluid, are considered by the evolution of the solute partial molar volume, which is a macroscopic data. The large volume contraction is interpreted according to the solvent structure and the intermolecular forces. In the case of an attractive mixture, the addition of a solute causes a change in the solvent organization and generates the aggregation of solvent molecules close to the solute (Fig. 2b). This phenomenon (which means the aggregation of solvent molecules around the solute) cannot be independent of local fluctuations of density.

Specific properties of supercritical fluids such as their low viscosity (matrix penetration as gas-like) and their relatively high density (solute solubilization as liquidlike) allow, for example, easier mass transfer which leads to their development on an industrial scale for extraction process. Concerning hydrothermal oxidation for waste management, the main interest is connected to the specific properties of supercritical water regarding its chemical reactivity with a high solubility of organic compounds and oxygen (suppression of interface) and a high reactivity (total organic destruction) [*4]. This paper is focused on recent work for materials processing in supercritical media related to fine particle synthesis, porous materials synthesis and modification, polymer synthesis and surface modifications.

\section{Fine particle synthesis}

Particle processing is today one of the major developments of supercritical fluid applications in industrial fields such as the chemistry, pharmaceutical, cosmetic and agro-food industries. Two main processes of fine particle synthesis in supercritical fluids have been developed. For the first one, particle formation is based on a physical transformation and for the second, particle formation is based on a chemical reaction. Two recent papers $\left[* * 5,{ }^{*} 6\right]$ present a good overview of the state of the art concerning the first kind of processes: (i) rapid expansion of supercritical solutions (RESS) characterized by a rapid depressurisation of a supercritical fluid solution containing the solute; the decompression being the driving force of particle nucleation and growth; (ii) supercritical anti-solvent (SAS) described by the dissolution of a supercritical fluid (generally $\mathrm{CO}_{2}$ ) in an organic solution containing the solute, the very low solubility of the solute in the resulting fluid leads to the particle nucleation and growth; (iii) particles from gassaturated solutions (PGSS) based on the depressurisation of a mixture consisting of a dense gas dissolved into a liquid, which can be either a solution (sometimes a suspension) of a compound or a melted solid; this depressurisation leads to the generation of particles. Numerous research studies are published and/or patented every year on these technologies; industrial applications are expected soon in many sectors, especially in the pharmaceutical industry.

Over the past decade, the second family of processes using supercritical fluids as reaction media to synthesize a number of finely divided materials has received considerable attention [**7]. Beyond the environmental aspect, this new way of materials processing is developed to give a better control of particle synthesis in terms of size, size distribution, morphology, chemical composition and structure. The main processes of fine particle synthesis by chemical methods, especially fine metal, semiconductor, nitride and oxide particles are presented hereafter. 


\subsection{Fine metal and semiconductor particle synthesis}

The thermal decomposition of bis(hexafluoroacetylacetonate)copper(II) hydrate in a supercritical $\mathrm{CO}_{2} /$ ethanol (80/20 wt.\%) mixture, at $19 \mathrm{MPa}$ and $200{ }^{\circ} \mathrm{C}$, leads to the synthesis of nanostructured copper particles $[8, * * 9]$. Different kinds of metal particles in terms of size, morphology and surface properties can be obtained (Fig. 3). The thermal decomposition of the metal precursor in a supercritical medium leads to a high nucleation rate, and thus, to the generation of metal clusters composed by few atoms. These clusters grow by binary contact and coalescence to give nanoparticles of a few nanometers (Fig. 3, step 1). As a function of the main process parameters (pressure, temperature, residence time, metal precursor concentration, ...), these nanoparticles can: (i) grow to larger crystalline particles (Fig. 3 , step 2; Fig. 4); (ii) aggregate to produce nanostructured particles with sizes between a few nanometers and a few micrometers (Fig. 3, step 3); (iii) be stabilized by surfactants, dendrimers, hyperbranched molecules, in order to give stabilized metal nanoparticles of a few nanometers (Fig. 3, step 4); (iv) be deposited into porous materials such as aerogels or membranes (Fig. 3, step 5). So, this process based on the thermal decomposition of metal precursors in supercritical media allows the synthesis of metal particles with control of the size (from few nanometers to few micrometers), the morphology and the surface properties, and recovery of the particles as dry powders without any trace of solvent.

Another approach inspired by solution chemistry concerns the use of reverse micelles in supercritical fluids as "nanoreactors" to synthesize metal and semiconductor nanoparticles [*10]. Production of silver and copper nanoparticles with water in sc-alkane microemulsions has been investigated. To replace flammable and expensive alkane solvents, reverse micelles in supercritical $\mathrm{CO}_{2}\left(\mathrm{sc}-\mathrm{CO}_{2}\right)$ were studied to synthesize metal $(\mathrm{Ag}, \mathrm{Cu})$ or semiconductor $(\mathrm{CdS})$ nanoparticles $[11, * * 12,13]$. Although the size and morphology of nanoparticles can be readily controlled by the reverse micelle properties, it is nevertheless difficult to collect the as-prepared nanoparticles owing to their aggregation and coalescence upon removal of the supercritical fluid. So, the reduction of metal precursors in $\mathrm{sc}-\mathrm{CO}_{2}$ in the presence of fluorinated surfactants to synthesize stabilized $\mathrm{Ag}, \mathrm{Ir}, \mathrm{Pt}, \mathrm{Pd}$ nanoparticles has been performed $\left[14,{ }^{*} 15\right]$. In the same way, stabilized copper nanoparticles were produced in $\mathrm{sc}^{-} \mathrm{H}_{2} \mathrm{O}$ [16].

\subsection{Fine nitride particle synthesis}

Fine nitride particles exhibit interesting properties in many fields such as chemistry, physics, mechanics and optics. Whereas the standard solid-state chemistry methods of nitride processing use very high pressures

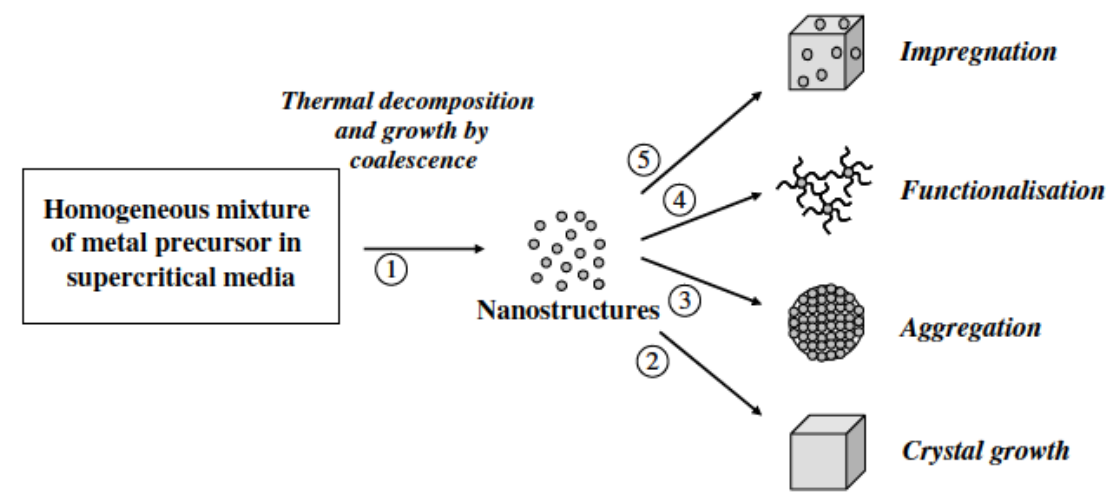

Fig. 3. Schematic representation of particles processing in supercritical media by thermal decomposition of metal precursors.
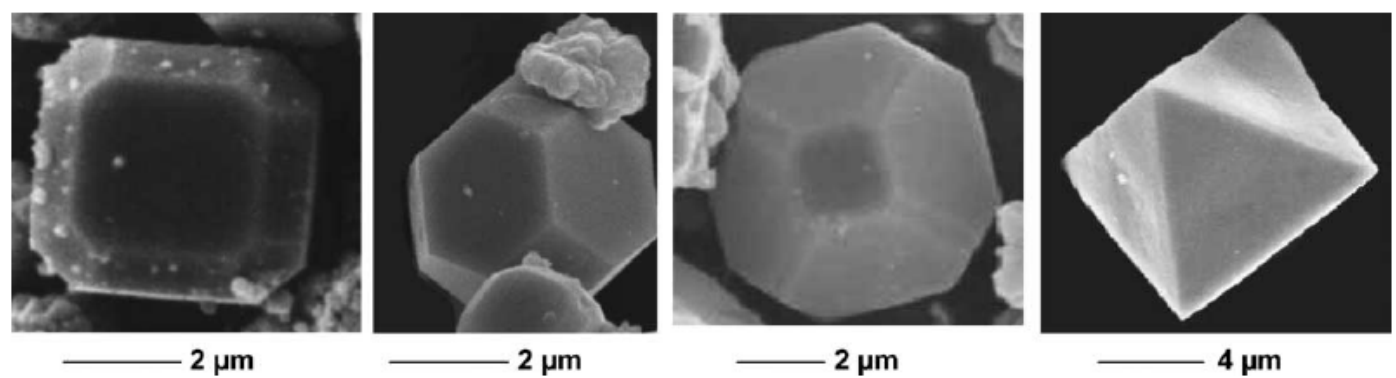

Fig. 4. SEM pictures of different shapes of crystalline particles of copper obtained by thermal decomposition of bis(hexafluoroacetylaceto nate)copper in a mixture of $\mathrm{CO}_{2} /$ ethanol $(80 / 20)$ at $200{ }^{\circ} \mathrm{C}$ and $19 \mathrm{MPa}\left[{ }^{* *} 9\right]$. 


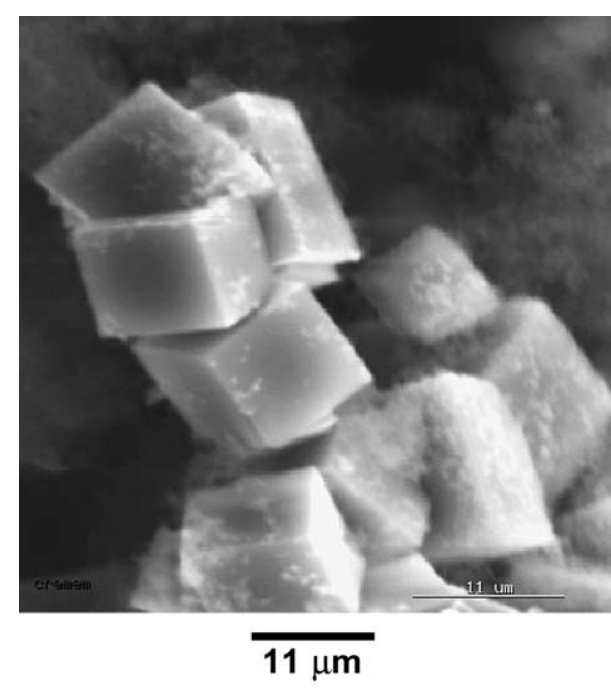

Fig. 5. SEM picture of cubic copper nitride monocrystals obtained by thermal decomposition of bis(hexafluoroacetylacetonate)copper in a mixture of $\mathrm{NH}_{3} /$ methanol $(70 / 30)$ at $200{ }^{\circ} \mathrm{C}$ and $16 \mathrm{MPa}$ [*17].

and/or temperatures, the process based on the thermal decomposition of metal precursors allows synthesis of nitride particles in supercritical ammonia at low temperature and low pressure [*17]. $\mathrm{Ni}_{3} \mathrm{~N}, \mathrm{Cu}_{3} \mathrm{~N}, \mathrm{Co}_{2} \mathrm{~N}$, $\mathrm{Cr}_{2} \mathrm{~N}, \mathrm{Fe}_{4} \mathrm{~N}$ particles were obtained by decomposition of the corresponding metal precursors in sc- $\mathrm{NH}_{3} /$ methanol (70/30) in a range of temperatures between 170 and $290{ }^{\circ} \mathrm{C}$ at $16 \mathrm{MPa}$. The nitride particle characteristics (size, size distribution, morphology, structure, surface properties) can be tuned with the process operating parameters. Fig. 5 presents, as example, SEM picture of cubic copper nitride monocrystals obtained by thermal decomposition of bis(hexafluoroacetylacetonate)copper in a mixture of $\mathrm{NH}_{3} /$ methanol $(70 / 30)$ at $200{ }^{\circ} \mathrm{C}$ and $16 \mathrm{MPa}\left[{ }^{*} 17\right]$.

\subsection{Fine oxide particle synthesis}

Since the beginning of the 1990s, supercritical fluids are widely used as reaction media for the synthesis of ceramics and closely related oxide materials, in order to improve the physicochemical properties of the powders. The process based on the thermal decomposition of a metal precursor is also very interesting to produce fine oxide particle with a control of the size, the size distribution, the morphology and the structure by tuning the process operating parameters $[* * 7,8, * * 9, * 17,18]$. Different kinds of fine oxide particles, nanocrystalline or amorphous, were obtained including $\mathrm{Fe}_{2} \mathrm{O}_{3}, \mathrm{Cu}_{2} \mathrm{O}$, $\mathrm{Cr}_{2} \mathrm{O}_{3}, \mathrm{Al}_{2} \mathrm{O}_{3}$ or $\mathrm{Ga}_{2} \mathrm{O}_{3}$ from the thermal decomposition of the corresponding metal precursors in sc- $\left(\mathrm{CO}_{2} /\right.$ ethanol) in a range of temperatures between 180 and $260{ }^{\circ} \mathrm{C}$ at about $16 \mathrm{MPa}$ (Fig. 6).

Different research teams using supercritical media to synthesize fine crystalline oxide particles have adapted

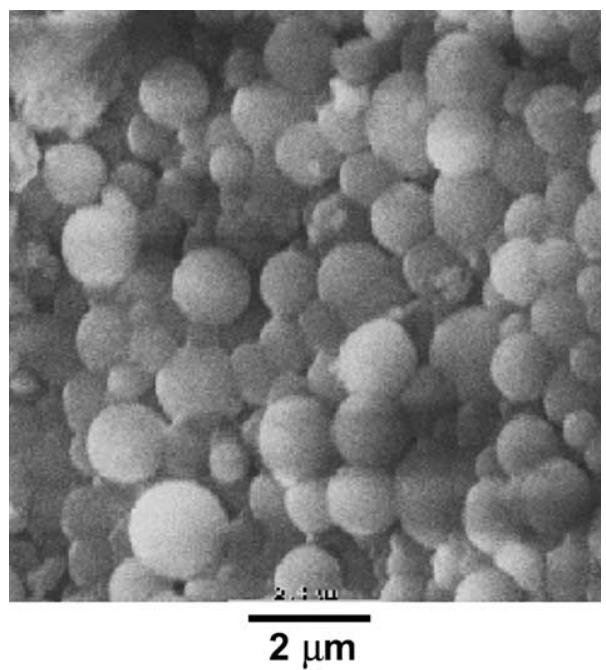

Fig. 6. SEM picture of alumina oxide particles obtained by thermal decomposition of bis(hexafluoroacetylacetonate)alumina in a mixture of $\mathrm{CO}_{2} /$ ethanol $(80 / 20)$ at $230{ }^{\circ} \mathrm{C}$ and $16 \mathrm{MPa}$ [*17].

the standard sol gel chemistry. Synthesis of $\mathrm{TiO}_{2}$ (anatase structure) or bimetallic oxides such as $\mathrm{MgAl}_{2} \mathrm{O}_{4}$ (spinel structure) and $\mathrm{BaTiO}_{3}$ (cubic structure) in scalcohol or sc- $\left(\mathrm{CO}_{2} /\right.$ alcohol $)$ has been investigated [19]. As in a previous process, a dry powder can be recovered by removal of the supercritical solution.

Reverse micelles in supercritical fluids as "nanoreactors" to produce oxides in a controlled way were also studied. For example, fine $\mathrm{TiO}_{2}$ particles were obtained with water in sc- $\mathrm{CO}_{2}$ reverse micelles [20].

Finally, hydrothermal synthesis is also a route to produce fine oxide particles in sub- and supercritical water. For example, hydrolysis followed by a dehydration reaction in sc-water of metal nitrates leads to a wide variety of metal oxide crystals [21]. In the same way, metal-organic precursors lead to metal oxide nanoparticles [22].

The synthesis of materials in supercritical fluids by a chemical process can be governed by high nucleation rate and low crystal growth as a function of the process operating parameters. So nanocrystals of a few nanometers are easily obtained. This process is very efficient to control the size, the morphology and the structure but also to synthesize fine metal, nitride and oxide particles at relatively low temperatures.

The research efforts are more and more focused on the stabilization of nanomaterials due to their very interesting properties, which can be different of those of bulk materials.

\section{Porous materials synthesis and modification}

Porous materials have attractive physicochemical properties due to their high surface areas and their 
porous structures. They have found a wide variety of applications in some areas such as catalysis, optics, phonic and thermal insulator. For example, porous materials with well-defined pore geometry are very interesting as catalysts and adsorbents for selective reactions. So, many investigations have been conducted to synthesize ordered porous materials and also to change porous structures by coating or loading them with functional molecules. As supercritical fluids have a high diffusivity, a low viscosity and a controllable solubility, they are good candidates for synthesizing porous structures and for coating on porous substrates.

\subsection{Aerogels}

A sol gel method is often used to synthesize porous materials. Classically, during the solvent vaporization step, the collapse of the three-dimensional network leads to a xerogel (Fig. 7). This collapse leads to the emergence of cracks or to the destruction of the material. In order to avoid this collapse, supercritical drying has been performed (Fig. 7) in numerous studies [23 25]. Recently, an organic aerogel of 2,3-didecyloxyanthracene (DDOA) was synthesized and dried using sc- $\mathrm{CO}_{2}$ as a solvent [26]. The formation of DDOA aerogel requires both higher pressure and temperature than for a classical supercritical drying process in order to obtain the $\mathrm{CO}_{2}$ solvation properties. But aerogels formed with this entire supercritical procedure looked like cotton material with a very low density of $2 \times 10^{-3} \mathrm{~g} \mathrm{~cm}^{-3}$. Silica aerogel microparticles were also easily obtained by a one step and versatile method with supercritical acetone as the solvent [27]. This method reduces the number of steps of traditional sol gel processes. Fiber-like structures and spherical morphologies were obtained. The properties of the resulting materials widely depend on the experimental parameters.

\subsection{Three-dimensional devices}

Mesoporous materials, such as silicates or aluminosilicates, have a great specific surface, a perfectly controlled porosity and unidirectional pores. They are distinguished from zeolites by pores which are definitely larger (from 1.8 to $15 \mathrm{~nm}$ as compared with a maximum of $1.3 \mathrm{~nm}$ for zeolites). These materials allow widening of the field of heterogeneous catalysis or separation of bulky molecules. Mesoporous materials can also act as nanoreactors. Coating with silica in supercritical fluid and in liquid ethanol onto nanoporous structures such as micro- and mesoporous silica and activated carbon has been investigated [28]. The silica precursor (tetraethylorthosilicate) dissolved in the liquid solvent does not enter into the pores due to the low diffusivity, the high velocity, the disturbance of the wettability and of the surface tension of the liquid solvent. On the other hand, a high diffusivity, a low viscosity and a noncohesiveness of supercritical fluids facilitate the introduction of the precursor more deeply into the pores than for the liquid solvent. Using the same idea, dimensionally ordered semiconductor nanowires of silicon were synthesized with the supercritical fluid inclusion-phase
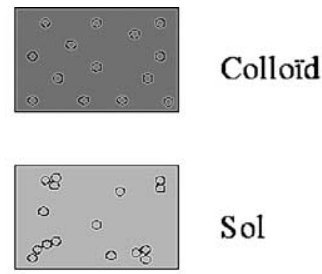

Sol

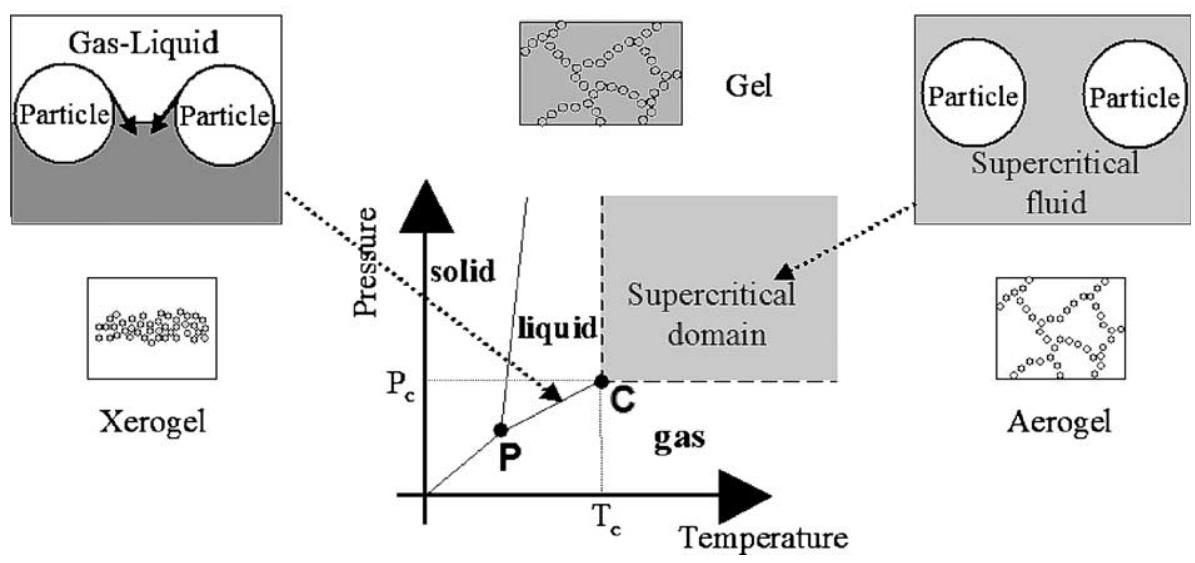

Fig. 7. Schematic representation of gel drying to produce xerogel and aerogel in a diphasic medium (gas liquid) and monophasic medium (supercritical fluid), respectively. 
technique [**12]. Metal and semiconductor nanowires were prepared within the pores of mesoporous silica hosts. This procedure is reproducible, extremely fast (15 30 min against days usually), efficient and cost effective. This inclusion technique is easily applied to other semiconductor and metal nanowires such as germanium, copper, cobalt, iron oxide and nickel. Another application of these interesting properties of supercritical fluids deals with the impregnation of polymeric substrates with antimicrobial substances such as silver, zinc and/or copper or metal-complexes or metal salts [29]. In this case, in addition to their interesting properties of diffusivity, viscosity and non-cohesiveness, supercritical fluids were chosen for their innocuousness compared with potentially toxic organic liquids.

Silica aerogels are also very porous materials with a large surface area. The pore size $(1 \quad 100 \mathrm{~nm})$ and porosity can be controlled by the preparation conditions. This large surface area enables the aerogel to retain a large number of nanoparticles. The key point is to fix them firmly on the silica structure. Incorporation of metal nanoparticles in silica aerogel was performed [30]. Dodecanethiol-passivated gold nanoparticles were prepared and a wet gel was then immersed in the particle solution leading to the formation of an ultra-fine metal particle/wet gel composite. The gel was then dried in sc$\mathrm{CO}_{2}$ in order to avoid shrinkage of the gel.

\subsection{Membrane}

$\mathrm{Al}_{2} \mathrm{O}_{3}$ membranes were directly modified by $\mathrm{TiO}_{2}$ deposition on ceramic substrates by hydrolytic decomposition of titanium tetraisopropoxyde in supercritical propan-2-ol [31]. Depending on the experimental conditions, different thickness (1 $3 \mu \mathrm{m})$, infiltration depths $(1530 \mu \mathrm{m})$ and pore sizes (micro- or mesopores) were obtained. Another recent procedure for ceramic membrane synthesis was a sol gel process carried out directly in supercritical carbon dioxide [**32]. This method was applied to the preparation of titania membranes on a macroporous $\mathrm{Al}_{2} \mathrm{O}_{3}$ support. The modification of experimental parameters allows control of the structure and the morphology of the membranes. In particular, both pressure and residence time strongly influence particle sizes and related surface areas. Mixed oxides were also obtained in sc- $\mathrm{CO}_{2}$. The synthesis of crystalline $\mathrm{Ce}_{1-x} \mathrm{Gd}_{x} \mathrm{O}_{2-x / 2}$ membranes was carried out in sc$\mathrm{CO}_{2}$ from cerium and gadolinium acetates as precursors [33].

\section{Polymer synthesis}

Since the late 1990s, chemical reactions performed in supercritical fluids and all subsequent related studies have increased exponentially [**34,**35]. This tremen- dous growth relies on the physicochemical properties of these fluids, which make them extremely attractive as green solvents. Among the supercritical fluids, $\mathrm{CO}_{2}$ is the most desirable for polymerization reactions. A large part of the studies revolve around homogeneous and heterogeneous radical polymerization techniques. Regarding ionic cationic and anionic and transition metal-catalyzed processes, recent results have shown these to be promising for the polymerization of vinylic, cyclic or olefinic monomers. Emulsion-templated polymers using a high internal phase $\mathrm{CO}_{2}$-in-water emulsion have been developed [*36].

Regarding anionic polymerization, copolymerization of epoxides with $\mathrm{CO}_{2}$ by species such as $\mathrm{Zn}(\beta$-diimine)X $\left(\mathrm{X}=\mathrm{Et}, \mathrm{OAc}, \mathrm{N}\left(\mathrm{SiMe}_{3}\right)_{2}, \mathrm{OH}, \mathrm{OMe}, \mathrm{O}^{i} \mathrm{Pr}, \mathrm{Br}, \mathrm{Cl}\right)$ was further investigated [37]. A pseudo-anionic ring opening polymerization of $\varepsilon$-caprolactone initiated by $\mathrm{M}\left(\mathrm{O}^{i} \mathrm{Pr}\right)_{3}$ $(\mathrm{M}=\mathrm{Al}, \mathrm{Y}, \mathrm{La})$ was reported and investigations of the $\mathrm{CO}_{2} / \mathrm{M}\left(\mathrm{O}^{i} \mathrm{Pr}\right)_{3}$-initiator interactions by in situ IR spectroscopy confirmed the reversible formation of carbonate type dormant species of the three metals, Al, Y, and La [38].

Regarding electrochemical synthesis, conducting polypyrrole films were obtained in sc- $\mathrm{CO}_{2}(8.39 .7 \mathrm{MPa}$; $3843^{\circ} \mathrm{C}$ ). The characteristics of the polypyrrole films synthesized in sc- $\mathrm{CO}_{2}$ are comparable to those prepared electrochemically in aqueous $\mathrm{HCl}$ and non-aqueous acetonitrile solutions [39].

A great number of studies have revolved around both the evaluation of the solubility of different species in various polymerization media [40 42] and the design and the use of surfactants and/or fluorinated species [*43]. Indeed, $\mathrm{CO}_{2}$-soluble amphiphilic block copolymer surfactants, incorporating a $\mathrm{CO}_{2}$-philic fluorinated or siloxane components and a $\mathrm{CO}_{2}$-phobic hydrophilic or lipophilic components, as well as fluorinated substituted catalysts monomers or additives have been designed and appear as the key entities for successful polymerizations. Fluorocarbon solvents were used for synthesized crosslinked polymer microspheres by dispersion polymerization [44].

Finally, sc- $\mathrm{CO}_{2}$ as a porogen solvent has attracted much interest for materials synthesis with macroporous polymer beads by suspension polymerization using supercritical fluid as a "pressure-adjustable" [*45] medium and with macroporous polymer monoliths [46].

\section{Surface modifications}

The specific properties of supercritical fluids can be used to improve or to modify surfaces by coating in a controlled way or by cleaning. These two aspects of surface modification in supercritical fluids are described hereafter. 


\subsection{Surface modification by coating}

Beyond the production of fine particles, another application of the processes described in 2 concerns the surface modification by coating:

(i) Surface modification by chemical processes in supercritical fluids: over the past decade, numerous studies have involved the development of chemical fluid deposition (CFD) techniques and related technologies based on the reaction of a precursor in a supercritical solution. The thermal decomposition of metal precursors was applied to the coating of substrates by metal, oxide or nitride films, in particular the coating of fine particles, even with complex morphologies. New core-shell structures, as $\mathrm{Ni} / \mathrm{Cu}$ and $\mathrm{SmCo}_{5} / \mathrm{Cu}$, were synthesized to exhibit interesting properties, more particularly in the magnetic recording field [47]. Fig. 8 presents the main steps of this process with two SEM pictures of a $\mathrm{Ni}$ particle before and after coating by a $\mathrm{Cu}$ shell. Another approach consists of the formation of metal, metal oxide or metal sulphide films onto substrate surfaces initiated by a reaction with a precursor in supercritical fluid (generally a reduction reaction with $\mathrm{H}_{2}$ ). For example, this process succeeded in depositing continuous palladium films at controlled depths within porous alumina disk [48]. Beyond the CFD techniques, a supercritical fluid immersion deposition (SFID) method was recently developed for producing thin films of metals and alloys on substrates or into porous solids of silicon, germanium, and other "fluorine-philic" elemental semiconductors [49]. $\mathrm{Pd}, \mathrm{Cu}, \mathrm{Ag}$ and other metal films were deposited on Si-based substrates in sc- $\mathrm{CO}_{2}$.

These methods for coating in supercritical fluids were developed to overcome the limitations of standard chemical vapour deposition process (CVD), as for example volatile, toxic and expensive precursors.

(ii) Surface modification by physical processes in supercritical fluids: RESS, SAS, and derivatives as well as PGSS are equally applied as microencapsulation and coating processes, in particular for microencapsulating active substance into a polymeric matrix. This includes the controlled drug release implicated in several industrial applications such as: pharmaceutics, agrochemistry, cosmetics, inks, paints etc. Among the numerous works published and/or patented in this field, two examples are discussed in this review: the fluidized bed coating technology in supercritical fluids as an experimental and promising method and a process of microencapsulation of protein particles within lipids using a novel supercritical fluid coating approach, as a process in an industrial development state. The fluidized bed coating technology is based on the RESS process. The coating material is dissolved in sc- $\mathrm{CO}_{2}$ and sprayed into the bulk of a fluidized bed containing the particles to coat. The first studies were applied for the coating of catalyst particles in a fluidized bed operating under atmospheric conditions. Recently, studies were performed on the coating of particles in a high-pressure fluidized bed $[* 50]$. This coating process is very promising because it couples the advantages of two wellknown processes, the RESS process and fluidized bed technology. But one of its limitations could be the coating of very fine particles and more precisely the stabilization in the fluidized bed of particle sizes lower than a few microns. The second example refers to a novel supercritical fluid way to process pharmaceutical products [51,**52]. It consists of the dissolution of one or more coating materials in sc- $\mathrm{CO}_{2}$ and then adjusting

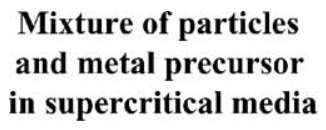

\section{Coated particles}

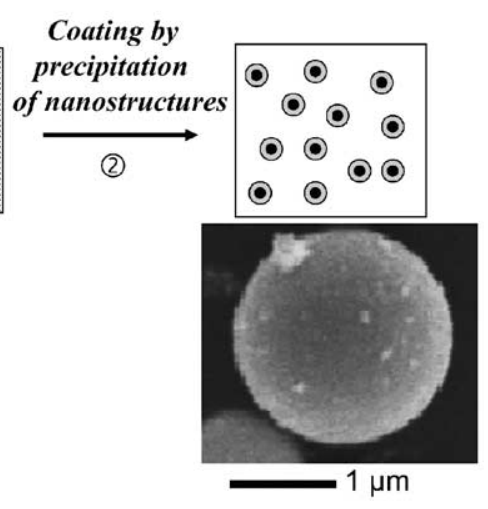

$\mathrm{Ni}$ particle coated by $\mathrm{Cu}$

Fig. 8. Schematic representation of particle coating in supercritical media by thermal decomposition of metal precursors. 
temperature and pressure working conditions in the reactor so that the coating material becomes insoluble and goes onto the surface of the dispersed particles. Today many efforts are provided in order to scale-up this process at the industrial scale under pharmaceutical conditions as good manufacturing practice (GMP).

\subsection{Surface modification by cleaning}

Supercritical carbon dioxide offers environmental and cost benefits to the semiconductor industry. Wet-chemical stripping processes are reaching their theoretical limits as circuit line architecture gets smaller [*53]. Compared to $\mathrm{CO}_{2}$ pellet cleaning or $\mathrm{CO}_{2}$ snow cleaning, sc- $\mathrm{CO}_{2}$ rapidly penetrates crevices and boundary layers where snow cannot [**54]. Low viscosity, low surface tension and the relatively high density of supercritical fluids aid in penetrating materials and removing contaminants. Moreover, initial cleaning of wafers has been suggested since solubility of non-polar organic compounds from greases, oils, lubricants and fingerprints is high in sc- $\mathrm{CO}_{2}$. More recently, new formulations and methods for removing unwanted materials from semiconductor wafers or other substrates have been proposed. These formulations may include co-solvent, surfactants, chelating agents and/or chemical reactants added to the supercritical fluid [55]. The efficiency of sc- $\mathrm{CO}_{2}$ cleaning of $\mathrm{Si}$ wafers has been developed at an industrial scale and the efficiency reaches $99.7 \%$ in $5 \mathrm{~min}$ [56]. Sc-ozone was also used to clean semiconductor wafers [57]. The combination of supercritical fluid with megasonic acoustic wave was recently used for cleaning wafers, substrates and semiconductor work pieces [58]. Another application of materials cleaning has been developed for the extraction of gadolinium from a mixture of $\mathrm{Gd}_{2} \mathrm{O}_{3}$ and $\mathrm{ZrO}_{2}$ selectively with sc- $\mathrm{CO}_{2}$ containing TBH $\mathrm{HNO}_{3}$ complex (TBH is tri- $n$-butyl phosphate) [59]. This method would be useful to the selective cleaning of materials contaminated with radioactive oxides such as $\mathrm{UO}_{2} / \mathrm{PuO}_{2}$. Even in the medical field, supercritical fluids have been used to clean implantable medical devices [60].

\section{Conclusions and perspectives}

Supercritical media present very attractive properties from a scientific point of view due to the possibility to modify continuously the properties of reaction media from a polar solvent to a non-polar solvent for water, for example, by adjustment of pressure and/or temperature. So, these media exhibit unique properties for well understanding kinetics and chemical pathways. The most promising developments of supercritical technologies, in the field of materials, will be processing of fine powders and core-shell particles, processing and/or impregnation of aerogels, processing of polymers and surface modifications.

From an industrial point of view, the developments of processes using supercritical fluid as reactive media are connected with the synthesis of materials with unusual physicochemical properties, which cannot be obtained with standard processes. Supercritical carbon dioxide is the most widely used supercritical medium in the recent literature. It is often chosen because of its particular characteristics such as its inocuity, its low cost and its easy using conditions.

The main limiting factors are the mass and heat transfers, which are generally very low. The hydrodynamic and the heat transfers are well depicted with commercial calculation programs but a large amount of work is still needed on the development of programs to predict the solubility of solutes and to model their reactivity in supercritical media. Furthermore, financial, legislative and psychological aspects are determining factors from a socio-economic point of view.

The technological transfer from laboratory to industry is promoted in the Community Initiative Programme INTERREG III B with the network "Supercritical Fluids and Materials Network SUPERMAT" in which research and innovation are fundamental prerequisites to combine economic growth and sustainable development [61].

\section{References}

The papers of particular interest have been highlighted as:

* of special interest;

** of very special interest.

[*1] Bonnaudin N, Cansell F, Fouassier O, editors. Supercritical fluids and materials. ISASF; 2003. ISBN 2905267399.

[*2] Arai Y, Sako T, Takebayashi Y, editors. Supercritical fluids. Springer series in materials processing. New York: Springer; 2003. ISBN 3540412484.

[*3] Sun Y P, editor. Supercritical fluid technology in materials science and engineering. New York Basel: Marcel Dekker; 2003. ISBN $082470651 \mathrm{X}$.

[*4] Bottreau M. Hydrothermal oxidation: a new concept for treatment of industrial and urban liquid wastes. In: Bonnaudin N, Cansell F, Fouassier O, editors. Supercritical fluids and materials. ISASF; 2003. p. 369 84. ISBN 2905267399.

[**5] Fages J. Powder processing using supercritical fluids. In: Bonnaudin N, Cansell F, Fouassier O, editors. Supercritical fluids and materials. ISASF; 2003. p. 33 85. ISBN 290526739 9.

[*6] Jung J, Perrut M. Particle design using supercritical fluids: literature and patent survey. J Supercrit Fluids 2001;20:179 219.

[**7] Loppinet Serani A, Aymonier C, Desmoulins Krawiec S, Etourneau J, Cansell F. Material synthesis using supercritical fluids as reactive media. In: Bonnaudin N, Cansell F, Fouassier O, editors. Supercritical fluids and materials. ISASF; 2003. p. 1 32. ISBN 2905267399. 
[8] Garriga R, Pessey V, Weill F, Chevalier B, Etourneau J, Cansell F. Kinetic study of chemical transformation in supercritical media of bis(hexafluoroacetylacetonate)copper(II) hydrate. J Supercrit Fluids 2001;20:55 63.

[**9] Pessey V, Garriga R, Weill F, Chevalier B, Etourneau J, Cansell F. Control of particle growth by chemical transformation in supercritical $\mathrm{CO}_{2}$ /ethanol mixtures. J Mater Chem 2002;12 95865.

[*10] Ye X, Wai CM. Making nanomaterials in supercritical fluids: a review. J Chem Ed 2003;80:198 204.

[11] Chandler McLeod M, McHenry RS, Beckman EJ, Roberts CB. Synthesis and stabilization of silver metallic nanoparti cles and premetallic intermediates in perfluoropolyether/ $\mathrm{CO}_{2}$ reverse micelle systems. J Phys Chem B 2003;107:2693 700 .

[**12] Holmes JD, Lyons DM, Ziegler KJ. Supercritical fluid synthesis of metal and semiconductor nanomaterials. Chem Eur J 2003;9:2144 50.

[13] Zhang J, Han B, Liu J, Zhang X, Liu Z, He J. Supercritical fluid synthesis of metal and semiconductor nanomaterials. Chem Commun 2001:2724 5.

[14] Shah PS, Husain S, Johnston KP, Korgel BA. Nanocrystal arrested precipitation in supercritical carbon dioxide. J Phys Chem B 2001;105:9433 40

[*15] Kameo A, Yoshimura T, Esumi K. Preparation of noble metal nanoparticles in supercritical carbon dioxide. Coll Surf A 2003;215:181 9.

[16] Ziegler KJ, Doty RC, Johnston KP, Korgel BA. Synthesis of organic monolayer stabilized copper nanocrystals in supercrit ical water. J Am Chem Soc 2001;123:7797 803.

[*17] Desmoulins Krawiec S, Aymonier C, Loppinet Serani A, Weill F, Gorsse S, Etourneau J, et al. Synthesis of nanostructured materials in supercritical ammonia: nitrides, metals and oxides. J Mater Chem 2004;14:228 32.

[18] Bobet JL, Desmoulins Krawiec S, Grigorova E, Cansell F, Chevalier B. Addition of nanosized $\mathrm{Cr}_{2} \mathrm{O}_{3}$ to magnesium for improvement of the hydrogen sorption properties. J Alloys Compd 2003;351:217 21.

[19] Bocquet JF, Chhor K, Pommier C. Barium titanate powders synthesis from solvothermal reaction and supercritical treat ment. Mater Chem Phys 1999;57:273 80.

[20] Stallings WE, Lamb HH. Synthesis of nanostructured titania powders via hydrolysis of titanium isopropoxide in supercritical carbon dioxide. Langmuir 2003;19:2989 94.

[21] Sue K, Murata K, Kimura K, Arai K. Continuous synthesis of zinc oxide nanoparticles in supercritical water. Green Chem 2003;5:659 62.

[22] Cabanas A, Poliakoff M. The continuous hydrothermal syn thesis of nanoparticulate ferrites in near critical and supercrit ical water. J Mater Chem 2001;11:1408 16.

[23] Li WC, Lu AH, Guo SC. Control of mesoporous structure of aerogels derived from cresol formaldehyde. J Coll Inter Sci 2002;254(1):153 7.

[24] Yim TJ, Kim SY, Yoo KP. Fabrication and thermophysical characterization of nano porous silica polyurethane hybrid aerogel by sol gel processing and supercritical solvent drying technique. Korean J Chem Eng 2002;19(1):159 66.

[25] Biesmans GLJG. Polyisocyanate based aerogel and their preparation. Patent WO 9844028.

[26] Placin F, Desvergne J P, Cansell F. Organic low molecular weight aerogel formed in supercritical fluids. J Mater Chem 2000;10:2147 9.

[27] Moner Girona M, Roig A, Molins E, Llibre J. Sol gel route to direct formation of silica aerogel microparticles using super critical solvents. J Sol Gel Sci Technol 2003;26:645 9.

[28] Wakayama H, Goto Y, Fukushima Y. A novel method for tailoring porous structures of nanoporous materials using supercritical solvents. Phys Chem Chem Phys 2003;5: 37848.

[29] Howdle SM, Bayston R, Webb PB. Impregnation of polymeric substrates with antimicrobial substances using supercritical fluids. WO03045448, 2003.

[30] Tai Y, Tajiri K, Watanabe M, Tanemura S. Method for incorporating metal nanoparticles in porous materials. US6569358, 2003.

[31] Brasseur Tilmant J, Pommier C, Chhor K. Synthesis of supported $\mathrm{TiO}_{2}$ membranes using supercritical alcohol. Mater Chem Phys 2000;64:156 65.

[**32] Guizard C, Julbe A, Rios GM, Sarrade S. Supercritical fluids and membrane technologies. In: Bonnaudin $\mathrm{N}$, Cansell $\mathrm{F}$, Fouassier O, editors. Supercritical fluids and materials. ISASF; 2003. p. 87 104. ISBN 2905267399.

[33] Sarrade S, Guizard C, Rios GM. New applications of super critical fluids and supercritical fluids processes in separation. Sep Purif Technol 2003;32:57 63.

[**34] Guillaume S. Polymer synthesis using supercritical fluids Part I. In: Bonnaudin N, Cansell F, Fouassier O, editors. Supercrit ical fluids and materials. ISASF; 2003. p. 105 36. ISBN 2 905267399

[**35] Cooper AI. Polymer synthesis using supercritical fluids Part II. In: Bonnaudin N, Cansell F, Fouassier O, editors. Super critical fluids and materials. ISASF; 2003. p. 137 49. ISBN 2 905267399

[*36] Butler R, Davies CM, Cooper AI. Emulsion templating using high internal phase supercritical fluid emulsions. Adv Mater 2001;13:1459 63

[37] Moore DR, Allen SD, Coates GW. Alternating copolymeriza tion of epoxides and carbon dioxides: advances in beta diiminate zinc catalysts. Polym Prepr (Am Chem Soc, Div Polym Chem) 2003;44:735 6 .

[38] Bergeot V, Tassaing T, Besnard M, Cansell F, Mingotaud A F. Anionic ring opening polymerization of $\varepsilon$ caprolactone in supercritical carbon dioxide: parameters influencing the reac tivity. J Supercrit Fluids 2004;28:249 61.

[39] Badlani RN, Mayer JL, Anderson PE, Mabrouk PA. Electro chemical synthesis and characterization of conducting poly pyrrole film in supercritical carbon dioxide. Polym Prepr (Am Chem Soc, Div Polym Chem) 2002;43:938 9.

[40] Drohmann C, Beckman EJ. Phase behavior of polymers containing ether groups in carbon dioxide. J Supercrit Fluids 2002;22:103 10.

[41] McHugh MA, Park I H, Reisinger JJ, Ren Y, Lodge TP, Hillmyer MA. Solubility of $\mathrm{CF}_{2}$ modified polybutadiene and polyisoprene in supercritical carbon dioxide. Macromolecules 2002;35:4653 7 .

[42] McHugh MA, Garach Domech A, Park I H, Li D, Barbu E, Graham P, et al. Impact of fluorination and side chain length on poly(methylpropenoxyalkylsiloxane) and poly(alkyl methac rylate) solubility in supercritical carbon dioxide. Macromole cules 2002;35:6479 82.

[*43] Wojcinski LM, Saraf MK, Charpentier P, DeSimone JM, Roberts GW. Polymerization of vinylidene fluoride in dense carbon dioxide. Polym Prepr (Am Chem Soc, Div Polym Chem) 2002;43:889.

[44] Coor SJ. 1,1,1,2 Tetrafluoroethane; from refrigerant and pro pellant to solvent. Fluorine Chem 2002;118:55 67 .

[*45] Rhor T, Knaus S, Gruber H, Sherrington DC. Preparation and porosity characterization of highly cross linked polymer resins derived from multifunctional (meth)acrylate monomers. Mac romolecules 2002;35:97 105 .

[46] Yu C, Xu MC, Svec F, Fréchet JMJ. Preparation of monolithic polymer with controlled porous properties for microfluidic chip applications using photoinitiated free radical polymerisation. Polym Sci Polym Chem 2002;40:755 69 . 
[47] Pessey V, Garriga R, Weill F, Chevalier B, Etourneau J, Cansell F. Core shell materials elaboration in supercritical mixture $\mathrm{CO}_{2}$ /ethanol. Ind Eng Chem Res 2000;39:4714 9.

[48] Fernandes NE, Fisher SM, Poshusta JC, Vlachos DG, Tsapat sis M, Watkins JJ. Reactive deposition of metal thin films within porous supports from supercritical fluids. Chem Mater 2001;13:2023 31

[49] Ye XR, Wai CM. Immersion deposition of metal films on silicon and germanium substrates in supercritical carbon dioxide. Chem Mater 2003;15:83 91.

[*50] Schreiber R, Vogt C, Werther J, Brunner G. Fluidized bed coating at supercritical fluid conditions. J Supercrit Fluids 2002;24:137 51 .

[51] Thies C, Ribeiro Dos Santos I, Richard J, Vandevelde V, Rolland H, Benoit JP. A supercritical fluid baud coating technology. 1 . process considerations. J Microencap 2003;20:87 96.

[**52] Deschamps FS, Richard J. In: Bonnaudin N, Cansell F, Fouassier O, editors. Supercritical fluids and materials. ISASF; 2003. p. 331 68. ISBN 2905267399.

[*53] McCoy M. Cleaner chemistry for cleaner chips. Chem Eng News 2001;79:10.
[**54] Weibel GL, Ober CK. An overview of supercritical $\mathrm{CO}_{2}$ applications in microelectronics processing. Microelectron Eng 2003;65:145 52.

[55] Xu C, Minsek DW, Roeder JF, Korzenski MB, Baum TH. Supercritical fluid cleaning of semiconductor substrates. US0125225, 2003.

[56] Recif Company, Perrut V. Available from: www.recif.com.

[57] Jackson D. Near critical and supercritical ozone substrate treatment and apparatus for same. WO11191, 2002.

[58] Rothman LB, Robey RJ, White R, Mount DJ, Farmer RB, Pope K. Supercritical fluid processes with megasonics. US0116176, 2003.

[59] Tomioka O, Enokida Y, Yamamoto I, Takahashi T. Cleaning of materials contaminated with metal oxides through supercrit ical fluid extraction with $\mathrm{CO}_{2}$ containing TBP. Prog Nucl Energy 2000;37:417 22.

[60] Pathak CP, Chinn JA, Thoma RJ. Cleaning of medical devices with supercritical fluids. US0021825, 2003.

[61] SUPERMAT, Fouassier O. Available from: www.univ pau.fr/ supermat. 